\title{
Mothers with post-traumatic stress disorder after traumatic childbirth struggled to survive and experienced nightmares, flashbacks, anger, anxiety, depression, and isolation
}

Beck CT. Post-traumatic stress disorder due to childbirth: the aftermath. Nurs Res 2004;53:216-24.

What is the essence of mothers' experiences of post-traumatic stress disorder (PTSD) after traumatic births?

DESIGN

Descriptive phenomenology.

\section{SETTING}

A website hosted in New Zealand.

\section{PARTICIPANTS}

38 mothers (mean age 33 y, 32\% primipara) from 4 countries (New Zealand, United States, Australia, and United Kingdom) who had experienced PTSD attributable to birth trauma (women reported that the diagnosis was made by a healthcare professional) and were willing to articulate their experiences. Length of time from birth trauma to study participation ranged from 6 weeks to 14 years.

\section{METHODS}

Women were recruited through Trauma and Birth Stress, a charitable trust in New Zealand founded to support women who had experienced birth trauma and to educate healthcare professionals and the general public about PTSD after childbirth. Women were asked to describe in writing their experiences of PTSD after childbirth in as much detail as they wished. 2 women hand wrote and sent their stories by post; 36 submitted their stories as email attachments. The phenomenological analysis involved reading and rereading participants' descriptions of PTSD after traumatic birth to describe the essence of the phenomenon, formulating the meanings of important statements pertaining directly to the experiences, and categorising the meanings into theme clusters. 9 participating mothers met with the researcher to validate preliminary findings, and the final results were reviewed on the internet by 4 mothers and 1 father.

\section{MAIN FINDINGS}

5 themes described the essence of the experience of PTSD after childbirth. (1) Going to the movies: please don't make me go! Mothers had flashbacks of the traumatic birth during the day and terrifying nightmares at night. These interfered with their relationships with their children and spouses. They felt that the memories were on automatic replay or endless loops. (2) A shadow of myself: too numb to try and change. Mothers considered themselves shadows of their former selves. They felt numb, detached, and devoid of emotion. For some mothers, these feelings began immediately after giving birth and continued after they were home. (3) Seeking to have questions answered and wanting to talk, talk, talk. Mothers had an intense need to know the details of their traumatic birth and obsessed over what had happened and why. Some women made repeated appointments with the healthcare providers who had done the delivery; others read obstetrical textbooks, and others wanted to revisit the delivery room. Women wanted to talk excessively about their experiences but found that healthcare providers, family, and friends became tired of listening. Some women then stopped discussing the birth and became withdrawn and separated from family and friends. Some women sought answers from higher levels such as the health and disability commissioner or state medical board; those who failed to get satisfaction in this quest often felt "retraumatised." (4) The dangerous trio of anger, anxiety, and depression: spiralling downward. Women experienced the emotions of anger, anxiety, and intense For correspondence: $\mathrm{Dr} \mathrm{C} \mathrm{T}$ Beck, University of Connecticut School of Nursing, Storrs, CT, USA. Cheryl.beck@uconn.edu

Source of funding: no external funding. depression. Anger was felt as rage and was directed at healthcare providers, family members, and self. Anxiety was experienced as panic attacks and manifested as chest pain, self inflicted wounds, and fear of sexual intercourse. Some women contemplated suicide. (5) Isolated from the world of motherhood: dreams shattered. Some women felt distanced from their infants and unable to feel any kind of closeness with them. Women also avoided other mothers and babies. Deciding whether to have another child was particularly distressing. Although some decided not to have another child, others prepared for another birth by preparing a detailed birth plan or asking specific questions when choosing a midwife.

\section{CONCLUSION}

The essence of mothers' experiences of post-traumatic stress disorder after traumatic births was characterised by daytime flashbacks or nightmares in which they relived the traumatic birth; feelings of numbness and detachment; an obsession with questions about what had happened and why and talking excessively about their experience; heightened feelings of anger, anxiety, and depression; and isolation from the world of motherhood.

\section{Commentary}

$B$ eck provides a compelling description of women's responses to traumatic deliveries, and poignantly describes the clinical presentation of PTSD in mothers after childbirth. A persuasive argument is presented that childbirth, for some women, may be experienced as an event beyond the range of usual human experience that results in the development of symptoms so severe it necessitates a PTSD diagnosis. This conclusion may challenge traditional beliefs about the types of events commonly identified as PTSD precursors.

Beck interviewed a large, diverse purposeful sample of women from 4 countries. Despite individual differences, the women described common experiences after birth, and the emergence of these core elements across the heterogeneous sample support data credibility. One limitation of the study is the reliance on maternal self report of a diagnosis of PTSD (reported to have been confirmed by a professional) as an inclusion criterion. Data for this study, in the form of written stories, were collected predominantly through email communications, an efficient and cost effective strategy for collecting data from widespread participants. Although mothers in the study found the process therapeutic, this form of electronic data collection is limited to individuals with well developed written verbal skills and access to appropriate technology.

The findings of this study permit privileged access to the emotions and experiences of women with PTSD after childbirth. As high levels of obstetrical interventions and maternal dissatisfaction with care received during labour and delivery are significantly associated with acute trauma symptoms, ${ }^{2}$ clinicians have a responsibility to provide supportive care and to be knowledgeable about the signs and symptoms of PTSD after childbirth. For women who perceive their delivery experiences as traumatic, opportunities to participate in debriefing sessions and to receive support and trauma counselling should be made available. In the postpartum period, nurses need to assess mothers who are at risk of PTSD for depression, isolation, and maternal-infant attachment problems and intervene appropriately.

Susan Jack, RN, PhD School of Nursing, McMaster University Hamilton, Ontario, Canada

1 Beck CT. Nurs Res 2004:53:28-35.

2 Creedy DK, Shochet IM, Horsfall J. Birth 2000;27:104-11. 